\title{
Evaluating Non-Interactive Domestic Situated SMS Messaging
}

John Downs

Department of Computer Science

University of Auckland

New Zealand

jdow038@aucklanduni.ac.nz

\section{Beryl Plimmer}

Department of Computer Science

University of Auckland

New Zealand

beryl@cs.auckland.ac.nz

\begin{abstract}
We present our evaluation of our SMS-to-photo-frame messaging system in diverse households. We explored whether low-cost, non-interactive ambient displays were useful when applied to domestic messaging. We performed an ethnographic study with two different types of households, during which we analysed their usage of the system and conducted a series of interviews. We found that all households used the system to some degree, but that the social context and communication styles were distinctly different between households comprised of families and those with fictive kin. This highlights the importance of considering the household structure when designing domestic technology. Additionally, our preliminary study explored the minimum requirements for a useful messaging appliance for the home, particularly with respect to interactivity.
\end{abstract}

\section{Keywords}

Ubiquitous computing, domestic messaging,

communication, situated display, mobile phone, digital photo frame, ethnographic evaluation study

Copyright is held by the author/owner(s).

CHI 2009, April 4 - April 9, 2009, Boston, MA, USA

ACM 978-1-60558-247-4/08/04.

\section{ACM Classification Keywords}

J.7. Computers in other systems: Consumer products.

H.5.2. User interfaces: Evaluation/methodology. 


\section{Introduction}

Situated displays have been proposed as a potential platform for communication and messaging in the home. Previous work has involved the construction of situated communication devices intended for domestic use. Situated messaging, also referred to as person-tolocation messaging, is one form of interpersonal communication. Instead of sending messages to a device that is specific to an individual, situated messaging enables messages to be left on shared displays that can be viewed by a number of people. In this way, messages can be broadcast to a group of people who share a physical space, such as a home. In addition, the physical location of such devices forms part of the messaging context. In many families, for instance, a great deal of intra-family communication occurs in the kitchen, and the refrigerator door has been established [6] as a common location for functional and sentimental family artefacts.

A number of studies have examined the development and evaluation of devices to support intra-family communication in various ways. TxtBoard [3] and HomeNote [5] were devices which received and displayed SMS messages from family members' mobile phones, the Whereabouts Clock [4] displayed family members' physical locations using a clock metaphor, and LINC [2] provided intra-family coordination and calendaring. In each of these studies, the authors conducted preliminary evaluation studies to determine the usefulness of the device. These studies focused exclusively on nuclear families as participating households. Families, however, are only one class of household. An increasing number of adults in the western world are living in non-traditional households such as single-person dwellings and childless couples.
In addition, shared living situations ('flats') are common with young adults and students. Sociologists and anthropologists recognise these household relationships as 'fictive kin' (e.g. [1]), supplementing or even supplanting traditional family relationships. It is important not to disregard these households, particularly since they are high users of digital communication technology.

These earlier studies have required the construction of custom technology, or the modification of relatively expensive devices such as tablet PCs. In our study we utilised digital photo frames as an inexpensive alternative. Newer photo frames support wireless networking, which allows a great deal of flexibility in the types of content that frames can display.

Current digital photo frame technology has some limitations, the most notable being non-interactivity. Previous studies on domestic situated displays have included some degree of interactivity to allow users to customise the display or to delete or navigate between messages. Applications that use digital photo frames as the display require a visual 'output-only' interface, without audio or interactive content. While some photo frames do have remote controls, buttons, or even touchscreens, these features are typically only available for navigation between the frame's menus and are not able to be used for communication with a separate computer system.

Our decision to use digital photo frames as the display for our messaging system allowed us to explore this restricted interaction. Unlike traditional desktop applications, which typically take advantage of rich interaction modalities, we instead used the opportunity 
to bring the level of interactivity back to a minimal baseline. From this baseline we hoped to ascertain whether interactivity is really required for messaging applications and for domestic technology generally, and if so, the extent and type of such interactivity.

In this study, we constructed a system to receive SMS messages from household members and display them on a digital photo frame. This system was designed to be used by both families and flats, and was evaluated with two households from each of these categories.

\section{Design and Implementation}

Messages are displayed on a Kodak EX1011 digital photo frame with a diagonal screen size of approximately 10 inches. Messages are routed via a PC which may be in a separate room. The frame and PC communicate wirelessly.

\section{System Architecture}

SMS messages are received through an Internet-hosted SMS gateway service. These messages are stored in a database on the PC. The PC also stores a series of background image files. When new messages are received the PC loads the background image files, adds the SMS messages, and constructs a new image file at the digital photo frame's native resolution. The photo frame wirelessly polls the PC for these images every 30 seconds. The system architecture is illustrated in Figure 1.

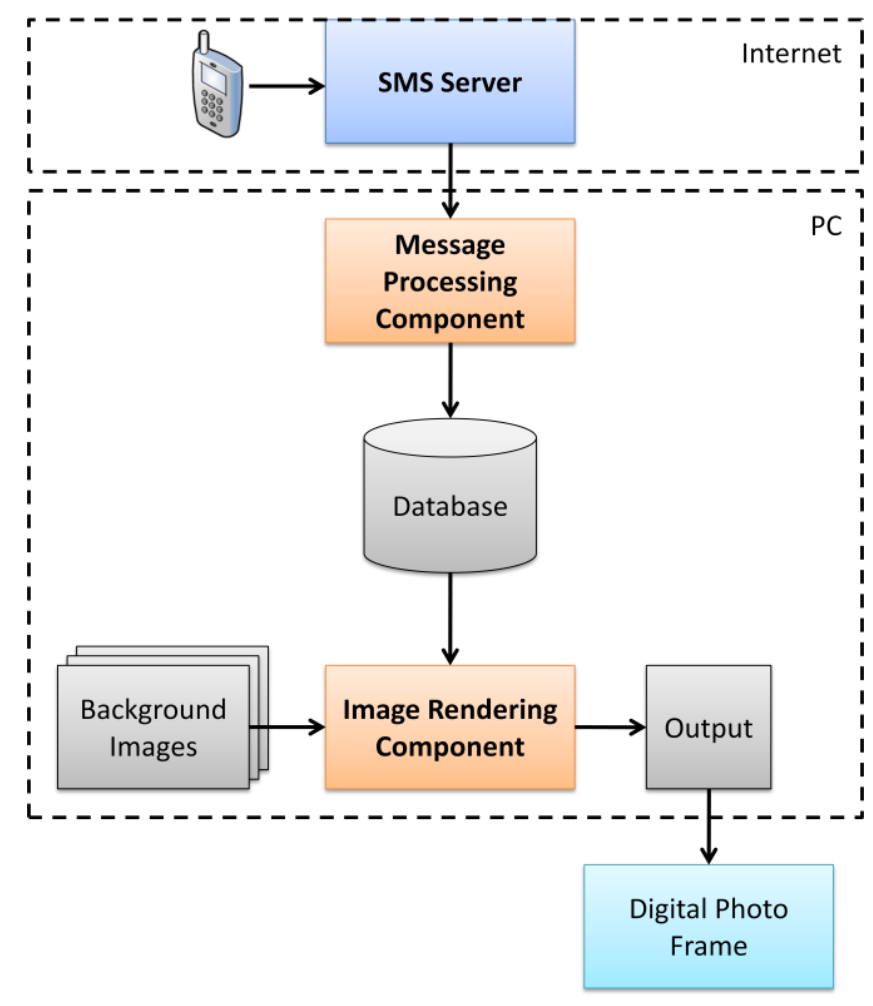

Figure 1. System architecture.

User Interface

The screen that is displayed on the photo frame (illustrated in Figure 2) consists of two panels: a photo panel and a message panel. The message panel (see Figure 2 callout) lists each SMS message in reverse chronological order, as well as the sender's name and the time the message was received. The most recent SMS message is colored red. As new messages arrive they displace any messages already on the frame. Due to the non-interactive nature of the device, there is no 


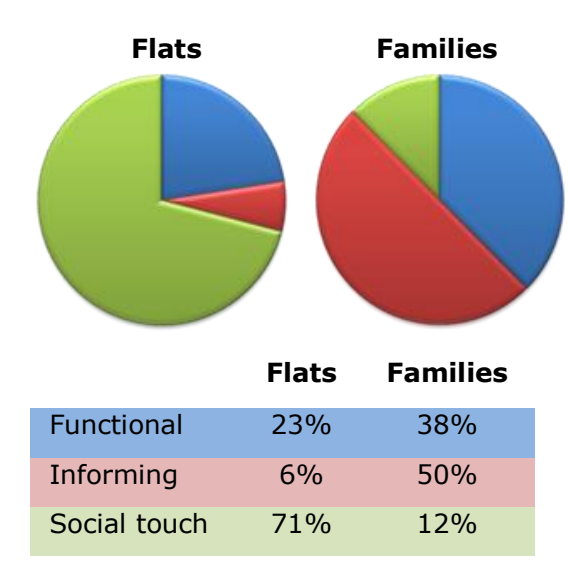

Figure 3. Messages by category for flats and families. way for users to scroll through messages or view old messages. However, users are able to visit a secure Web page on their computer that lists older messages sent to their household through the system.

\section{Evaluation Methodology}

We conducted an ethnographic evaluation study with four households (two families and two flats). Households were provided with the prototype system for approximately one month each. Within the two families the teenage children (ranging in age from 15 to 20 years) typically used their mobile phones more than their parents, although all family members used them to some extent, including for SMS messaging. In the two flats all household members ('flatmates', ranging in age from 19 to 24 years) used mobile phones on a regular basis, including regularly sending SMS messages to each another.

Household members were interviewed on three separate occasions. These interviews focused on the household members' communication patterns, use of the technology, and the content of the messages the household members were sending. Additionally, each message sent through the system was categorised by two people according to a taxonomy based on that used in [3]. We combined the categories "call to action" and "reminder" from [3] into a single "functional" category, as our initial attempts at coding suggested it was difficult to differentiate messages between these categories without a full knowledge of the context in which the message was sent. For example, a message such as "we have guests coming for dinner" could be interpreted as a reminder (if the household members were already aware of this), an implicit call to action (for example, to tidy the house or cook the meal), or with some other context-specific meaning. The three categories of messages in our taxonomy were:

- Functional: messages intended to support management of the household, often requesting assistance or action to be taken (such as "can someone pick me up?" or "please put the oven on"). These requests are typically intended for the entire household rather than a specific person.

- Informing, awareness, reassurance: messages intended to inform others about the sender's activities, but without necessarily requiring any action on the part of the sender (such as "I'll be home late").

- Social touch: messages intended to support intrahousehold social bonds, or for humorous or trivial purposes (such as "Thanks for dinner").

Once the messages were classified according to this taxonomy, the resulting data were used for preliminary quantitative analysis.

\section{Summary of Findings}

The households sent a combined total of approximately 100 messages to the system over the period of study.

Flats vs. Families

Figure 3 shows the proportion of messages by category for families and flats. Flats used the system mostly

(71\%) for social messaging. Most messages contained little practical content but were more in the form of social messages such as "I'm bored, gimmie gimmie gimmie". Flatmates rarely used the system for informational or awareness messaging, with some flatmates commenting that due to the informal organisational structure, the flatmates "[didn't] really care about knowing who's going to be home when". 
In contrast, families used the system mostly (50\%) for informational messages. Many of these messages were from the families' teenage children, informing their parents of their whereabouts (for example, "Coming home. I'll be there in about an hour.").

\section{Device Constraints}

All households noted that the non-interactivity of the device affected their use of the system in a variety of ways. Due to the inability to scroll through or delete messages on the digital photo frame, household members quickly discovered that frequent use of the device could result in messages being removed from the screen before they had a chance to view them. Based on usage volumes reported in previous studies (e.g. [5]) we had not expected this to be an issue. Interestingly, most participants commented that the inability to reply did not matter to them. In the event that they wanted to reply to the sender, they simply sent a normal SMS message on their own mobile phone, or contacted the sender by some other means.

Another limiting aspect of the device was its limited audio support. While the digital photo frame did contain a speaker for audio output, this speaker was not available during the photo slide show mode that we used to display the message content. Many households remarked that the lack of an audible alert when messages were received resulted in them missing the messages, possibly until it was too late to respond to them.

\section{Discussion and Future Work}

Our preliminary ethnographic study found that there were substantial differences between the way our technology was used by the two types of households.
In this study, we only examined two types of households, but there are many more (for example, single-person dwellings and childless couples). The results from this study have illustrated that we cannot assume the same communication patterns apply across all of these types of households. Household communication patterns for families (such as those described in [3] and [5]) did not hold true for flats. Accordingly, even within the realm of domestic computing, it is crucial to design technology with the specific type of household in mind.

This evaluation study was designed to test both the technology (i.e. digital photo frames) and the application (i.e. domestic SMS messaging). In particular, by using a completely non-interactive device, our aim was to explore the ways in which these photo frames could be repurposed as ambient displays for messaging purposes while maintaining their zerointeractivity characteristic.

The results of our study shows that this particular application may not be directly suitable for digital photo frame technology, due to the caveats of noninteractivity and lack of audio. Our interviews suggested that the participants would have used the system more had it included these features. However, the actual amount of interactivity that would be required to support this sort of domestic messaging is minimal. Interactivity is required in order to allow users to scroll through messages and delete them as appropriate. A small number of simple pushbuttons could be used to achieve such a goal. Most photo frames already have this hardware but do not directly expose it to third-party developers. By using a photo frame with an open API or replacing firmware it may be 
possible to repurpose these buttons. It would also be possible to have external pushbutton devices (perhaps connected wirelessly to the computer), or a simple custom device could be constructed that includes these buttons as well as a screen. Similarly, by adding an audio speaker, providing auditory notifications of new messages would be trivial.

Once this small amount of additional interactivity is available, the scope for additional applications of the technology increases. For example, such a system might be used for medication reminders for elderly house-bound people. During the day, an inexpensive photo frame could remind the person to take their required medication. Once the person has completed this task they could press the button and the notification would disappear. Such a system would also provide an opportunity for monitoring these patients. If a patient does not respond to an alert by pressing the appropriate button, this may be considered an irregularity and the system may take further action. This application, and many others, would be relatively simple to implement on our platform once some limited degree of system interactivity is available.

Even without this interactivity, digital photo frame technology could still be repurposed for use as domestic ambient displays, but the specific applications must be carefully considered to ensure they are satisfactory while being completely non-interactive. For example, a household may have a series of digital photo frames which contain fixed-size 'widgets' with content such as weather, news, traffic updates, and household members' whereabouts. Such photo frames could be placed at convenient locations around the home, and even without the interactivity we describe above, may still be useful as ambient awareness devices for these types of content.

Our future work will continue in the area of situated displays, and will explore the level and types of interactivity that different applications may require. By varying the amount and type of interactivity on situated displays, we can begin to understand how different applications of this technology present varying requirements, and how different types of households will integrate the technology into their routines.

\section{References}

[1] Gubrium, J. F., and Buckholdt, D. R. (1982). Fictive Family: Everyday Usage, Analytic, and Human Service Considerations. American Anthropologist 84:878-885.

[2] Neustaedter, C., \& Brush, A. J. B. (2006). "LINCing" the family: the participatory design of an inkable family calendar. Proc. CHI 2006.

[3] O'Hara, K., Harper, R., Unger, A., Wilkes, J. Sharpe, B., \& Jansen, M. (2005). TxtBoard: From text-to-person to text-to-home. Proc. CHI 2005.

[4] Sellen, A., Eardley, R., Izadi, S., \& Harper, R. (2006). The Whereabouts Clock: Early testing of a situated awareness device. Proc. CHI 2006.

[5] Sellen, A., Harper, R., Eardley, R., Izadi, S., Regan, T., Taylor, A. S., et al. (2006). HomeNote: Supporting situated messaging in the home. Proc. CSCW 2006.

[6] Swan, L., \& Taylor, A. S. (2005). Notes on fridge surfaces. Proc. Human Factors in Computing Systems 2005. 